\title{
Revista de Comunicación en España. Una aproximación histórica
}

\author{
Antonio Castillo-EsParcia \\ Universidad de Málaga \\ acastilloe@uma.es \\ Vicente PEÑa TIMÓN \\ Universidad de Málaga \\ sema@uma.es \\ Sebastián MAÑAS VALLE \\ Universidad de Málaga \\ vicente@uma.es
}

\begin{abstract}
Resumen:
En España, las revistas científicas han adquirido un peso importante en la actividad de difusión de la ciencia. Este texto persigue presentar el panorama actual de las revistas de comunicación en España y analizar su distribución geográfica, temática y temporal.

Con ello vamos a comprobar cómo se han incrementado las revistas en comunicación y cómo se han ido insertando en la actividad investigadora de las universidades. Para ello, se han analizado las principales revistas españolas en comunicación.
\end{abstract}

Palabras clave: Revista científicas; investigación; comunicación.

\section{Scientific journals of communication in Spain. A historical approach}

\begin{abstract}
:
In Spain, scientific journals have become an important weight in the activity of dissemination of science. This text aims to present the current landscape of communication scientific journal in Spain and analyze its thematic, geographical distribution and temporal.

Thus we will check how the scientific journal have increased communication and how have been inserting in the research activities of universities. Therefore we have analyzed the main Spanish journals in communication.
\end{abstract}

Key Words: Scientific journals; research; communication

Referencia normalizada:

Castillo-Esparcia, A., Peña Timón, V. y Mañas Valle, S. (2014): Revista de comunicación en España. Una aproximación histórica. Historia y Comunicación Social. Vol. 19. Núm. Especial Febrero. Págs. 621-630.

Sumario: 1.Introducción. 2. La revista científica. Una breve historia. 3. Revistas científicas e investigación en España. 4. Conclusiones. 


\section{Introducción}

Las revistas científicas surgen históricamente como necesidad de comunicación de los investigadores y para poder explicar y expandir sus resutados de investigación. La ciencia debe difundirse y los investigadores deben tener canales de expresión de sus hablasgos y resultados. Por tanto, el surgimiento en 1665 de la revista Le Journal de Savans es fruto de la necesidad de comunicación de los investigadores. Surgida como elemento de difusión, dio paso a una serie indefinida de publicaciones periódicas que pretendían ser expositores de la ciencia, de los investigadores y de las investigaciones.

Con el paso de los años, las revistas se han hecho más complejas, más periódicas, con procesos internos de revisión lo que ha motivado muchos cambios en el modo y manera de difundir la ciencia.

Hoy en día vivimos, participamos, de una auténtica eclosión de publicaciones científicas que abarcan publicaciones generalistas, especializadas, naciones o internaciones. Empero, este actual fértil campo de creación contrasta con las limitadas revistas en la historia de la comunicación en España.

El nacimiento de revistas científicas es la consecuencia de que existe la necesidad de que investigadores cuenten con un instrumento de expresión que llegue a la comunidad científica para poder difundir sus investigaciones y estudios. Sin embargo, ese hecho que debe ser fruto de la madurez intelectual de un determinado campo del conocimiento no se ha concitado en España sino que la aparición de revistas científicas ha sido fruto de iniciativas personales de investigadores y docentes sin ayuda de editoriales, centros de investigación o Universidades.

Con este texto se pretende aportar evidencias sobre el nacimiento de las revistas de comunicación, las motivaciones de sus editores, los espacios temáticos y geográficos que abarcaba y la necesidad de que la comunicación cuente con espacios científicos de difusión de la ciencia.

\section{La revista científica. Una breve historia}

Los inicios conceptuales de la revista, como texto reducido de explicación de los resultados de una investigación y que desea ser difundido a una determinada comunidad científica, se piensa que tuvo lugar cuando las principales academias científicas del siglo XVII adoptaron un sistema de comunicación entre pares, es decir específico para los científicos. Así, se estableció un correo de confianza a semejanza del sistema que ya utilizaban los diversos países europeos en sus comunicaciones diplomáticas u oficiales (Castillo y Carretón, 2010: 294).

Por ello siempre han existido mecanismos de información para conocer qué, quién, cómo y sobre qué se está investigando. En determinadas épocas los sistemas de 
comunicación humanos sólo permitían la creación de centros de estudios que reunían a los investigadores y que el conocimiento y las investigaciones se producían a través de las consultas personales o documentales en esos centros.

Con la irrupción de la imprenta se pudo realizar una creciente difusión de la investigación científica y el consecuente conocimiento. En ese proceso comenzaron a aparecer las revistas como instrumentos de difusión de las investigaciones. Y cuando las revistas aumentan en número se comienza a establecer la necesidad de que se valore para qué sirven, a quién llega y qué repercusión tienen los artículos en la comunidad científica. Incluso algunos investigadores han señalada que el trabajo científico y, por extensión, el concepto de científico es una actividad dinámica y sólo es ciencia aquello que se publica en los artículos científicos (Jiménez, 1992: 175).

Las universidades protagonizaban la enseñanza en una estricta jerarquía que reflejaba su origen dual griego y cristiano. El escalón más alto lo ocupaba la teología. En el segundo nivel se encuentran la lógica (racional) y la filosofía natural; los estudiantes medievales debían dominar ambas materias antes de que se les permitiera ingresar en la facultad de Teología, en las que pasaban años enfrascados en el estudio de textos en lugar de buscar la santidad. En la base de la pirámide se hallaban las diversas artes luberales, divididas en dos grupos denominados según las palabras latinas que significaban "cuatro" y "tres": el quadrivium de la astronomía, la geometría, la aritmética y la música, y el trívium de la retótica, la gramártica y la lógica (Fara, 2009: 119).

Las primeras apelaciones a realizar estudios sobre el impacto de las revistas como consecuencia de la importancia que le confieren por propios investigadores datan de los años 20. Son postulados que pretenden introducir determinados conceptos como son la relevancia de los artículos, la influencia de los textos entre los investigadores o la mejora del conocimiento de una disciplina. Así, Gross y Groos (1927) señalaron la necesidad de que los artículos científicos podían medir su influencia a partir de las citas de otros autores. Y eso suponía un criterio inicial de calidad científica que era otorgado por los propios pares al considerar que la investigación era relevante para una disciplina y que mejoraba su conocimiento

Sin embargo, los conflictos bélicos condicionaron sobremanera la expansión de los estudios al respecto. De ahí, que hasta mediados de los años 50 no se comience a realizar de una manera sistemática estudios bibliométricos. Esta disciplina se basa en la necesidad de que los investigadores reconozcan la labor investigadora de otras personas por lo que el aspecto esencial de la bibliometría es la citación, entendido como el reconocimiento intelectual de una investigación no sólo realizada sino publicada. Este es un elemento caudal para poder entender el panorama actual de la investigación ya que no sólo es importante realizar investigaciones sino que éstas deben estar a disposición de la comunidad científica, es decir, debe ser publicada. Eso ha llevado a que se establezcan mecanismos de conocimiento de las investigaciones mediante libro o revistas. La investigación se centra esencialmente en las revistas por lo que se establecen un listado de revistas que son más relevantes en un determinado campo de conocimiento. Por tanto, un primer aspecto que debe tenerse presente en 
la investigación son las bases de datos que se encuentran a disposición de los investigadores. Las bases son numerosas y sería pertinente establecer una jerarquía entre ellas porque numerosas factores determinan su presencia intelectual entre los investigadores como pueden ser:

- política científica de los países al primar unos índices frente a otros

- la política editorial que se inserta las grandes bases de datos de revistas

- la preponderancia de bases de datos de determinados ámbitos geográficos frente a otros

- la escasa presencia de determinados idiomas en algunas bases de datos

- la escasa actividad e iniciativa de revistas para ser incluidas en las bases de datos

Las principales leyes bibliométricas son:

1. Ley de crecimiento exponencial. La pregunta de investigación de De Solla Price consistía en conocer la naturaleza de los artículos científicos en el ámbito mundial. Desde la perspectiva de los autores, De Solla Price (1965: 515) señala que las publicaciones científicas se centran en unos pocos cientos de hombres ("from a study of the citations or journals by journals i come to the conclusion that most of these strips correspond to the work, of, at most, a few hundred men at any one time"). Para De Solla Price se produce una multiplicación cada 10-15 años

2. Ley de productividad de los autores. Lotka señaló que la relación entre trabajos e investigadores se desarrollan de una manera constante. Trata de localizar los autores más productivos. Ya en la década de los años 20, Lotka se planteaba esta cuestión al señalar que "it would be of interest to determine, if possible, the part which men of different calibre contribute to the progress os scienc" (1926. 317). Este estudio se realizó sobre las publicaciones en Resúmenes Químicos ("Chemical abstracts") en el período 1907-1916. Se constata que a medida que aumenta el número de trabajo disminuye el número de autores.

3. Ley de dispersión de la literatura científica. Formulada por Bradford establece que si consultamos literatura especializada sobre un tema determinado, encontramos que la mayor parte de trabajos sobre él se encuentran publicados en un pequeño número de revistas, denominado núcleo.

Para Castillo y Carretón (2010. 293) la bibliometría se ha insertado plenamente en el quehacer de los investigadores en comunicación y desempeña un apartado que afecta a diversos ámbitos de las publicaciones científicas:

(...)los estudios bibliométricos permiten conocer los ámbitos en los que se desarrollan las temáticas de un campo científico, conocer las tendencias de las investigaciones, identificar a los grupos de investigación (investigadores) que están trabajando, verificar el grado de interconexiones internacionales entre investigaciones4, la relación entre género, entre investigadores, los sistemas de citas de otras publicacio- 
nes, las autocitas de las publicaciones, las autocitas de los investigadores, los centros de investigación en los que se realizan (educativos, profesionales, investigadores). Es decir, todo un conjunto de parámetros que permiten establecer un panorama del estudio de la investigación en un campo concreto.

\section{Revistas científicas e investigación en España}

El bagaje sobre publicaciones de revistas ya comienza a contar con una serie de fuentes que nos permiten conocer el panorama investigador en España. El papel de las revistas incluidas en el JCR soslaya a la mayoría de las revistas de comunicación españolas y se centra en aquella que provienen del ámbito anlosajón (Castillo-Esparcia, Moraga y Almansa, 2012: 251)

El campo de la comunicación abarca dos grandes áreas de conocimiento, como son Comunicación Audiovisual y Publicidad, y Periodismo. Es un campo científico de amplio espectro y que cuenta con una cantidad apreciable de docentes e investigadores pero que, todavía, no dispone del suficiente y necesario número de revistas. En este sentido, la presencia de revistas en IN-RECS -comparado con otros ámbitos científicos- es aún reducido (Cuadro 1). En este cuadro se observa que el campo de la comunicación es el que en IN-RECS cuenta con menor número de revistas.

Cuadro 1.- Revistas en Ciencias Sociales

\begin{tabular}{|c|c|c|}
\hline CAMPO DE CONOCIMIENTO & $\begin{array}{c}\text { NÚMERO DE } \\
\text { REVISTAS }\end{array}$ & $\begin{array}{c}\text { NÚMERO REVISTAS } \\
\text { FUENTE }\end{array}$ \\
\hline ANTROPOLOGÍA & 46 & 9 \\
\hline DOCUMENTACIÓN & 33 & 8 \\
\hline $\begin{array}{c}\text { CIENCIA POLÍTICA Y DE LA } \\
\text { ADMINISTRACIÓN }\end{array}$ & 59 & 7 \\
\hline COMUNICACIÓN & 24 & 32 \\
\hline ECONOMÍA & 136 & 32 \\
\hline EDUCACIÓN & 166 & 13 \\
\hline GEOGRAFÍA & 51 & 28 \\
\hline PSICOLOGÍA & 108 & 16 \\
\hline SOCIOLOGÍA & 82 & 6 \\
\hline URBANISMO & 43 & 1 \\
\hline
\end{tabular}

Fuente: elaboración propia

Otro aspecto relevante para calcular el factor de impacto son las denominadas revistas fuente, tal como se refleja también en el cuadro 1. IN-RECS emplea cuatro criterios para definir las revistas fuente:

- Pervivencia o antigüedad. 
- Calidad editorial, entendida ésta en una doble vertiente: calidad en el proceso editorial (empleo de un riguroso sistema de selección y evaluación de originales) y reputación y prestigio del editor y comités editoriales.

- Impacto científico de las revistas españolas de Ciencias Sociales, tanto a nivel internacional como nacional.

- Opinión de los investigadores españoles, medida a través de encuestas sobre la calidad percibida de las revistas de su especialidad.

Los dos primeros criterios actúan como prerrequisito para la entrada en la base de datos, esto es, aquellas revistas que no los cumplen quedan automáticamente descartadas; los dos segundos, son los criterios definitivos para la selección.

En la actualidad, se encuentran en IN-RECS un total de 24 revistas en comunicación que abarcan a revistas generalistas y revistas especializadas (cuadro 2).

Cuadro 2.- Revistas de Comunicación en IN-RECS

\begin{tabular}{|c|c|c|}
\hline NOMBRE REVISTA & $\begin{array}{c}\text { AÑO } \\
\text { CREACIÓN }\end{array}$ & EDITOR \\
\hline $\begin{array}{c}\text { Revista Latina de } \\
\text { Comunicación Social }\end{array}$ & 1998 & $\begin{array}{c}\text { Laboratorio de Tecnologías de la Información } \\
\text { y Nuevos Análisis de Comunicación Social }\end{array}$ \\
\hline Comunicación y Sociedad & 1998 & $\begin{array}{c}\text { Facultad de comunicación (Universidad de } \\
\text { Navarra) }\end{array}$ \\
\hline Comunicar & 1993 & Grupo Comunicar \\
\hline $\begin{array}{c}\text { Estudios sobre el Mensaje } \\
\text { Periodístico }\end{array}$ & 1994 & Facultad de Ciencias Sociales y de la \\
Comunicación
\end{tabular}




\begin{tabular}{|c|c|c|}
\hline Questiones Publicitarias. & 1993 & $\begin{array}{c}\text { Grupo de Investigación Método, Análisis y } \\
\text { Estrategias de la Comunicación Empresarial e } \\
\text { Institucional (Univ. De Sevilla) }\end{array}$ \\
\hline $\begin{array}{c}\text { Historia y Comunicación } \\
\text { Social }\end{array}$ & 1996 & $\begin{array}{c}\text { Departamento de Historia de la Comunicación } \\
\text { Social (Universidad Complutense de Madrid) }\end{array}$ \\
\hline Icono 14 & 2003 & $\begin{array}{c}\text { Grupo de Investigación Icono 14 (Universidad } \\
\text { Complutense de Madrid) }\end{array}$ \\
\hline $\begin{array}{c}\text { CIC. Cuadernos } \\
\text { de Información y } \\
\text { Comunicación }\end{array}$ & 1995 & Universidad Complutense de Madrid \\
\hline Comunicación y Hombre. & 2005 & Universidad Fco. De Victoria \\
\hline Coneixement i Societat & 2003 & Generalitat de Catalunya \\
\hline $\begin{array}{c}\text { I/C Revista científica } \\
\text { de información y } \\
\text { comunicación }\end{array}$ & 2003 & $\begin{array}{c}\text { Departamento de Periodismo (Universidad de } \\
\text { Sevilla) }\end{array}$ \\
\hline $\begin{array}{c}\text { Mediaciones Sociales } \\
\text { Pensar la publicidad }\end{array}$ & 2007 & $\begin{array}{c}\text { Grupo de Investigación Identidades Sociales y } \\
\text { Comunicación (Universidad Complutense de } \\
\text { Madrid) }\end{array}$ \\
\hline Revista de la SEECI & 1998 & $\begin{array}{c}\text { Departamento de Comunicación Audiovisual } \\
\text { y Publicidad II (Universidad Complutense de } \\
\text { Madrid) }\end{array}$ \\
\hline Vivat Academia & 1998 & $\begin{array}{c}\text { Departamento de Comunicación Audiovisual } \\
\text { y Publicidad II (Universidad Complutense de } \\
\text { Madrid) }\end{array}$ \\
\hline
\end{tabular}

Fuente: elaboración propia

Cuando se establece la necesidad de que los procesos editoriales de las revistas sean progresivamente profesionalizados, nos encontramos con una situación en la que las revistas son editadas, en un inmensa mayoría, por las universidades con un porcentaje del 91,6\% sobre el total de las revistas que aparecen en IN-RECS. Únicamente aparece una revista (Telos) que es editada por una organización empresarial como es la Fundación Telefónica (cuadro 3). Todo ello contrasta con el sistema de edición de las revistas de comunicación incluidas en el JCR, que son editadas por grandes grupos editoriales.

Cuadro 3.- Tipo entidad editora

\begin{tabular}{|c|c|c|}
\hline UNIVERSIDADES & \multicolumn{2}{|c|}{$\mathbf{2 2}(\mathbf{9 1 , 6 \% )}$} \\
\hline Empresas & 1 & $4,16 \%$ \\
\hline Generalitat de Cataluña & 1 & $4,16 \%$ \\
\hline
\end{tabular}

Fuente: elaboración propia

Y dentro de las universidades, como entidades editoras, nos encontramos con que un tercio de las revistas son editadas por la Universidad Complutense de Madrid $(33,3 \%)$ y, en segundo lugar, la Universidad de Sevilla con una 16,6\%. Entre estas dos universidades públicas españolas alcanzan el 50\% de las revistas de comunicación incluidas en IN-RECS. 
Cuadro 4.- Principales universidades editoras de revistas

\begin{tabular}{|c|c|c|}
\hline UNIVERSIDAD & \multicolumn{2}{|c|}{ NÚMERO DE REVISTAS } \\
\hline Universidad Complutense de Madrid & 8 & $33,3 \%$ \\
\hline Universidad de Sevilla & 4 & $16,6 \%$ \\
\hline
\end{tabular}

Fuente: elaboración propia

Cuando se analiza la titularidad de las universidades, se observa que las universidades públicas editan el 70,8\% de las revistas, siendo un 20,8\% editadas por Universidades privadas (cuadro 5).

Cuadro 5.- Titularidad universidad

\begin{tabular}{|c|c|c|}
\hline TIPO DE UNIVERSIDAD & $\mathbf{N}^{\mathbf{0}}$ & $\%$ \\
\hline Universidad Pública & 17 & $70,8 \%$ \\
\hline Universidad Privada & 5 & $20,8 \%$ \\
\hline \multicolumn{2}{|c|}{ Fuente: elaboración propia }
\end{tabular}

Sobre los años de creación de las revistas, se constata que los años 80 fueron los inicios de las principales revistas españolas en comunicación, aunque el verdadero incremento se produce en los años 90, cuando aparecen el 45,8\% de las revistas analizadas (cuadro 6). Es, sin duda, la década de las 90 el inicio de la consolidación del proceso investigador una vez que los centros, en los que se imparte comunicación, han asentado los planes de estudio y se han iniciado por programas de doctorado y los necesarios grupos de investigación.

Cuadro 6.- Año de creación de las revistas

\begin{tabular}{|c|c|c|}
\hline AÑO & $\mathbf{N}^{\mathbf{N}}$ & $\%$ \\
\hline Años 80 & 2 & $8,3 \%$ \\
\hline Años 90 & 11 & $45,8 \%$ \\
\hline 2000 -2005 & 9 & $37,5 \%$ \\
\hline 2005 -actualidad & 2 & $8,3 \%$ \\
\hline
\end{tabular}

Fuente: elaboración propia

Seguidamente, en el primer quinquenio del año 2000, se crea el otro gran grupo de revistas científicas, con un $37,5 \%$ de las actuales. Todo este proceso nos permite señalar que la vida útil de las revistas científicas en España ha sido reciente, ya que el $83 \%$ de las revistas tiene escasos veinte años y su consolidación ha sido difícil por el amateurismo de sus creadores, por las escasez de recursos económicos y por los limitados procesos bibliométricos.

Este incremento del papel de las revistas científicas como vehículo de expresión de la investigación se ha visto motivado por los requisitos exigidos por instancias educativas de evaluación de la actividad del profesorado universitario. En este sentido, la Comisión Nacional de la Actividad Investigadora (CNEAI) y la Agencia Nacional de Evaluación de la Calidad (ANECA) han transformado el papel de las revistas 
debido a la justificación de los méritos de investigación del profesorado universitario (Castillo-Esparcia, 2011: 131)..

Tal como se establece en el documento de Principios y Orientaciones para la aplicación de los criterios de evaluación de la Agencia Nacional de Evaluación de la Calidad y Acreditación (ANECA): "En cualquier caso, se valoran preferentemente las aportaciones que sean artículos en revistas de reconocido prestigio, aceptándose como tales las que ocupen posiciones relevantes en los listados por ámbitos científicos en el "Subject Category Listing" del Journal Citation Reports del Science Citation Index (SCI), del Social Sciences Citation Index (SSCI) y del Arts and Humanities Citation Index (AHCI) (Institute of Scientific Information, -ISI- Philadelphia, PA, USA), en el Philosopher's Index, en el Répertoire Bibliographique de Louvain o similares".

\section{Conclusiones}

Las revistas científicas han venido adquiriendo una mayor presencia en el ecosistema investigador español. En el campo de la Comunicación se ha producido un incremento significativo de la cantidad y calidad de las revistas científicas debido a que desde instancias educativas se exige resultados de investigación, que son medidos por el tipo de revista en la que se publican los resultados de los estudios.

Los investigadores disponen de mejores revistas que exigen mejores artículos. Ese binomío, en principio adecuado, hace que numerosos artículos sean rechazados por las revistas a pesar de que disponen de una adecuada calidad. La razón es que los autores se centran en aquellas revistas que cuentan con mejores valoraciones en las bases de datos que valorizan las revistas científicas. Y son muchos artículos para un número reducido de revistas.

\section{Bibliografía}

CASTILLO, A. Y CARRETÓN, M.C. (2010): "Investigación en Comunicación. Estudio bibliométrico de las Revistas de Comunicación en España", en Revista Comunicación y Sociedad, XXIII (2), Pamplona, p. 289-327.

CASTILLO-ESPARCIA, A., RUBIO-MORAGA, A. Y ALMANSA-MARTÍNEZ, A. (2012): "La Investigación en comunicación. Análisis bibliométrico de las revistas de mayor impacto del ISI", en Revista Latina de Comunicación Social, 67. La Laguna (Tenerife): Universidad de La Laguna, p. 248-270 [Recuperado el 22 de octubre de 2013]

CASTILLO-ESPARCIA, A. (2011): "El rol de las publicaciones científicas en Comunicación en el EEES: indexación e impacto", en Revista internacional de 
Relaciones Públicas, Málaga: Instituto de Investigación en Relaciones Públicas, p. 125-154. Málaga: Instituto de Investigación en Relaciones Públicas, p. 51-72. [Recuperado el 23 de octubre de 2013]

FARA, P. (2009): Breve historia de la Ciencia, Ariel. Madrid.

GROSS, P. Y GROSS, E. (1927): "College libraries and chemical education", en Science, 66: 385-389.

JIMENEZ CONTRERAS, E. (1992): "Las revistas científicas: el centro y la periferia", en Revista Española de Documentación Científica, 15, 2, p. 174-182.

PARK, H. W., \& LEYDESDORF, L. (2008): "Knowledge linkage structures in communication studies using citation analysis among communication journals", Scientometrics, Vol. 75, No. 3, p. 439-462. 